
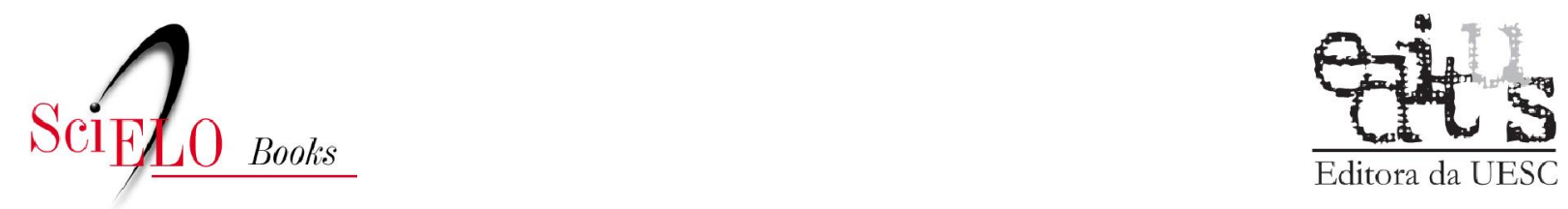

Editora da UESC

\title{
22 - Estudos biogeográficos sobre o gênero Thaumatomyrmex Mayr, 1887 (Ponerinae, Ponerini)
}

\author{
Benoit Jahyny \\ Hilda Susele Rodrigues Alves \\ Dominique Fresneau \\ Jacques H. C. Delabie
}

\section{SciELO Books / SciELO Livros / SciELO Libros}

JAHYNY, B., et al. Estudos biogeográficos sobre o gênero Thaumatomyrmex Mayr, 1887 (Ponerinae, Ponerini). In: DELABIE, JHC., et al., orgs. As formigas poneromorfas do Brasil [online]. Ilhéus, BA: Editus, 2015, pp. 327-343. ISBN 978-85-7455-441-9. Available from SciELO Books $<$ http://books.scielo.org $>$.

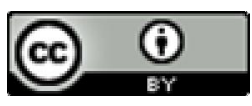

All the contents of this work, except where otherwise noted, is licensed under a Creative Commons Attribution 4.0 International license.

Todo o conteúdo deste trabalho, exceto quando houver ressalva, é publicado sob a licença Creative Commons Atribição 4.0.

Todo el contenido de esta obra, excepto donde se indique lo contrario, está bajo licencia de la licencia Creative Commons Reconocimento 4.0. 


\title{
Estudos biogeográficos sobre $\mathrm{o}$ gênero Thaumatomyrmex Mayr, 1887 (Ponerinae, Ponerini)
}

\author{
Benoit Jahyny, Hilda Susele Rodrigues Alves, \\ Dominique Fresneau, Jacques H. C. Delabie
}

\section{Resumo}

O gênero Thaumatomyrmex é endêmico da Região Neotropical e sua distribuição continental se estende desde o México até o Sul do Brasil e ao norte da Argentina, com presença em várias ilhas do Caribe. Suas espécies vivem em ambientes variados como florestas úmidas tropicais, áreas periodicamente inundadas ou ainda ambientes secos, tais como Cerrado ou Caatinga. Suas colônias, que possuem provavelmente a menor população entre os membros da família Formicidae, se instalam em cavidades naturais que ocorrem no solo, na serapilheira ou na base de árvores. Elas se alimentam de Penicillata vivos (Myriapoda, Diplopoda), que são suas presas quase exclusivas. As operárias são distintas das de outros Ponerinae principalmente pela sua morfologia cefálica característica. Suas mandíbulas são extremamente compridas, finas e arqueadas, com três dentes compridos, afunilados, pontudos e recurvados. Para o estudo biogeográfico, nos referimos ao agrupamento de espécies que Kempf definiu em 1975 a partir de critérios morfológicos, os grupos Cochlearis, Ferox e Mutilatus. As espécies do grupo Cochlearis vivem nas ilhas das Grandes Antilhas; as espécies do grupo Ferox têm a distribuição mais ampla, praticamente em toda a Região Neotropical, entre os limites de $18^{\circ} \mathrm{N}$ (México) e $19^{\circ} \mathrm{S}$ (Brasil), desde o nível do mar a mais de 2.000 metros de altitude; as espécies do grupo Mutilatus têm uma distribuição limitada à America do Sul (Brasil, Argentina e Paraguai), com ocorrência desde o nível do mar até $1.000 \mathrm{~m}$ de altitude. Existem várias combinações de pares simpátricos de espécies no gênero Thaumatomyrmex e um habitat não parece comportar simultaneamente mais do que duas espécies do gênero. As simpatrias conhecidas consistem sempre de uma espécie grande e de outra menor e, provavelmente por isso, permitem sempre a cada uma o acesso diferenciado aos recursos alimentares e aos lugares de nidificação. A diferença de tamanho é, muito provavelmente, um dos fatores que permitem a coexistência das espécies, reduzindo a sobreposição de seus respectivos nichos. A casta "gine" foi descrita apenas recentemente e gines de diversas espécies estão presentes em algumas coleções. Em espécies em que falta a casta morfologicamente distinta da gine, a reprodução ocorre por meio de gamergates. Atualmente são conhecidas menos de uma dúzia de espécimes de gines de poucas espécies de Thaumatomyrmex,

JAHYNY, Benoit; ALVES, Hilda Susele Rodrigues; FRESNEAU, Dominique; DELABIE; Jacques H. C. Estudos biogeográficos sobre o gênero Thaumatomyrmex Mayr, 1887 (Ponerinae, Ponerini). In: DELABIE, Jacques H. C. et al. As formigas poneromorfas do Brasil. Ilhéus: Editus, 2015. p. 327-343. 
todas pertencentes ao grupo Ferox, enquanto nos demais grupos (Cochlearis e Mutilatus) e a maioria dos táxons do grupo Ferox, as espécies se reproduzem através de gamergates ou inexiste informação sobre a existência de gine. Essa situação e a ampla distribuição latitudinal do grupo Ferox sugerem também que esse grupo (ou parte do mesmo) corresponde à linhagem mais basal do gênero. Uma vez que essas formigas possuem características muito peculiares de nidificação e alimentação com a baixa capacidade de dispersão das espécies onde as fêmeas aladas não existem, espera-se que as populações não respondam bem a mudanças climáticas, assim como a variações rápidas da qualidade de seu habitat. Thaumatomyrmex parece ser, assim, um modelo ideal para abordar estudos sobre a estrutura da biodiversidade neotropical e biomonitoramento.

\section{Abstract}

Biogeographic studies on the genus Thaumatomyrmex Mayr, 1887 (Ponerinae. Ponerini) - The genus Thaumatomyrmex is an endemic of the Neotropical Region, with a continental distribution that extends from Mexico to southern Brazil and northern Argentina, with an occurrence in several Caribbean islands. Its species live in several types of environment, such as tropical rainforests, periodically flooded areas or dry environments, such as savanna or semiarid vegetation. Their colonies, with probably the smallest populations in the whole Formicidae family, settle in natural cavities that occur in soil, litter or at trees bases. They feed on live Penicillata (Myriapoda, Diplopoda), which are almost their exclusive prey. The workers are distinct from other Ponerinae, mainly due to their characteristic cephalic morphology. Their jaws are extremely long, thin and arched, with three long, tapered, pointed and curved teeth. The gyne morphotype was recently described and gynes of several species are conserved in some collections. In species lacking the morphologically distinct gyne, reproduction occurs by means of gamergates. For this biogeographic review, we refer to the grouping of species that Kempf defined in 1975 from morphological criteria, being the Cochlearis, Ferox and Mutilatus groups. The species of the Cochlearis group live in the islands of the Greater Antilles; the species of the Ferox group have wider distribution, occurring practically throughout the Neotropics, in the range of $18^{\circ} \mathrm{N}$ (Mexico) to $19^{\circ} \mathrm{S}$ (Brazil), from the sea level to over 2,000 meters altitude; the species of the Mutilatus group have their distribution limited to South America (Brazil, Argentina and Paraguay), between $03^{\circ} \mathrm{S}$ and $29^{\circ} \mathrm{S}$, occurring from sea level up to $1,000 \mathrm{~m}$ altitude. There are several combinations of sympatric species pairs within this genus but one particular habitat does not seem to simultaneously support more than two species of the genus. Known sympatries always consist of a large species and another smaller one, which allows each of them differential access to food resources and nesting places. The size difference is, in fact, one of the factors that allow the species coexistence, reducing the overlap of their respective niches. Currently, less than a dozen specimens from a few species of Thaumatomyrmex are known from gynes, all of which belong to the Ferox group. In the other groups (Cochlearis and Mutilatus) and a majority of the taxa of the Ferox group, the species reproduce through gamergates or there is little information on the existence of gynes. This situation and the wide latitudinal distribution of the Ferox group also suggest that this group (or part of) is the most basal clade of the genus. Since these ants have very specific nesting and feeding characteristics, together with a low dispersion capacity for the species where winged females do not occur, it is expected that their populations would be severely impacted by climate changes and rapid alterations of their habitat quality. Thaumatomyrmex therefore seems then to be an ideal model for studies on the structure of Neotropical biodiversity and for biomonitoring. 


\section{Introdução}

Thaumatomyrmex Mayr, 1887 (Ponerinae, Ponerini) é um gênero endêmico da Região Neotropical, cuja distribuição continental se estende desde o México até o Sul do Brasil e ao norte da Argentina, com presença em várias ilhas do Caribe (KEMPF 1975; BARONI URBANI; DE ANDRADE, 2003; JAHYNY et al., 2008). Suas espécies vivem desde o nível do mar até $2.000 \mathrm{~m}$ de altitude e são encontradas em ambientes variados como florestas úmidas tropicais, áreas periodicamente inundadas ou ainda ambientes secos, tais como Cerrado ou Caatinga (JAHYNY et al., 2008, 2009). Este gênero de formiga de morfologia incomum (Figura 22.1) possui uma biologia muito peculiar (BRANDÃO et al., 1991; JAHYNY et al., 2008,2009 ). Suas colônias, que possuem provavelmente as menores populações entre os membros da família Formicidae (Figura 22.2), se instalam em cavidades naturais como conchas de gastrópodes, cupinzeiros, ninhos de vespas em terra ou, ainda, casulos de mariposas, que ocorrem no solo, na serapilheira ou na base de árvores (Figura 22.3) (JAHYNY et al., 2002, 2008, 2009; JAHYNY, 2010). Elas se alimentam de Penicillata vivos (Myriapoda, Diplopoda) (Figura 22.4), que são suas presas quase exclusivas (BRANDÃO et al., 1991; JAHYNY et al., 2008, 2009; JAHYNY, 2010; RABELING et al., 2012).

As espécies do gênero Thaumatomyrmex são conhecidas principalmente pela casta operária. A gine [alada e que perdeu as asas] foi descrita apenas recentemente (VAZQUEZ et al., 2010), apesar de sua existência ser conhecida há muito tempo (D.R. SMITH, in litt., In: KEMPF, 1975) e de gines de várias espécies estarem presentes em algumas coleções (B.J. obs. pess.). No entanto, a casta morfologicamente distinta da gine é desconhecida na maioria das espécies de Thaumatomyrmex, nas quais a reprodução ocorre por meio de operárias fecundadas (JAHYNY et al., 2002), chamadas "gamergates" sensu Peeters; Crewe (1984).

O desenvolvimento e o uso do extrator de Winkler como técnica de coleta de formigas permitiram a captura de uma quantidade razoável de indivíduos de Thaumatomyrmex em diversas partes da Região Neotropical. Esta técnica de amostragem da fauna da serapilheira é agora amplamente utilizada em larga escala em toda a Região Neotropical em estudos de ecologia de comunidades ou mesmo simplesmente para inventariar a fauna. Esta técnica é reconhecida como especialmente eficiente para a captura das formigas da serapilheira (BESTELMEYER et al. 2000; PARR; CHOWN 2001; LONGINO et al., 2002. UNDERWOOD; FISHER, 2006), das quais fazem parte as Thaumatomyrmex (DELABIE et al., 2000; DELABIE et al., 2007a).

A biogeografia das formigas neotropicais, ainda incipiente (KUSNEZOV, 1963; LATTKE, 2003), foi estudada, sobretudo, em nível regional (LATTKE, 2003, 2007) ou em relação a um determinado táxon ou grupo de espécies (DELABIE et al., 1997, 2007b, 2011). Por ser relativamente bem distribuído na Região Neotropical, o gênero

FIGURA 22.1 - Cabeça de uma operária de Thaumatomyrmex contumax (Ilhéus, BA) (Microscopia Eletrônica de Varredura, M.C. Malherbes, Laboratoire d'Ethologie Expérimentale et Comparée - LEEC, Universidade Paris XIII). Operária de T. contumax, Automontage

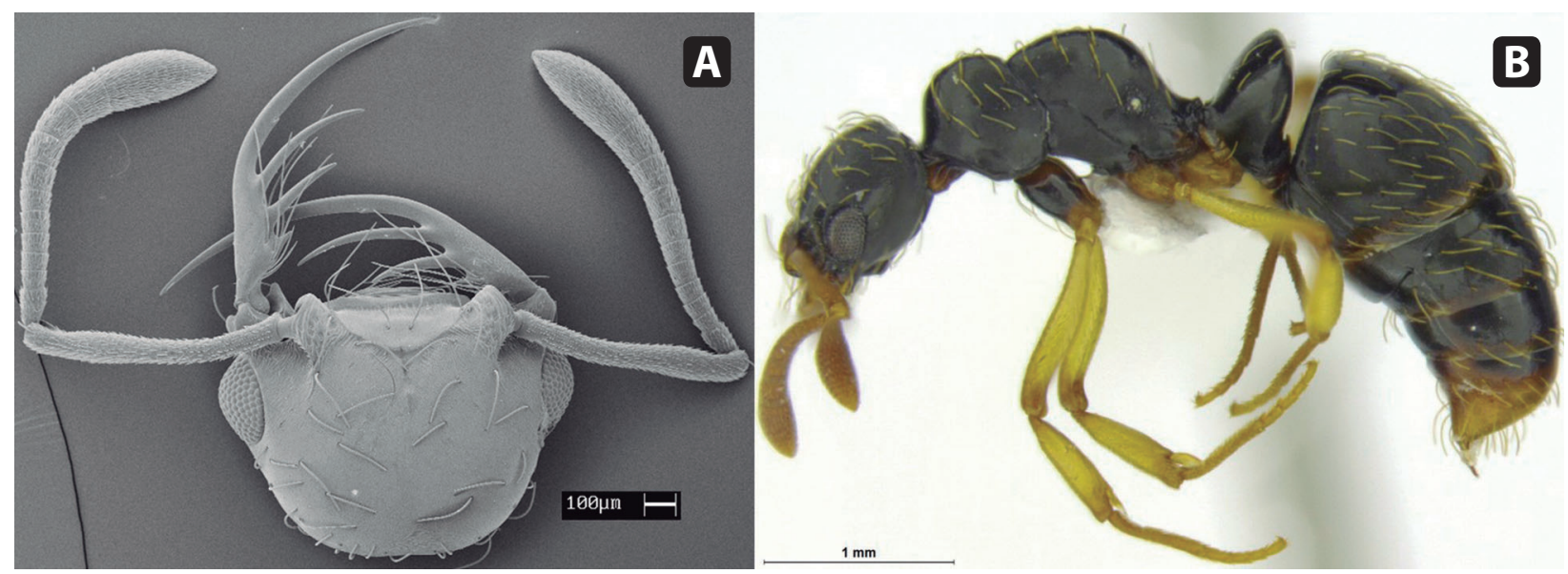


FIGURA 22.2 - População de uma colônia completa de Thaumatomyrmex sp.11 (3 fêmeas, 1 macho, 1 pupa, 2 larvas, 3 ovos), La Selva, Costa Rica

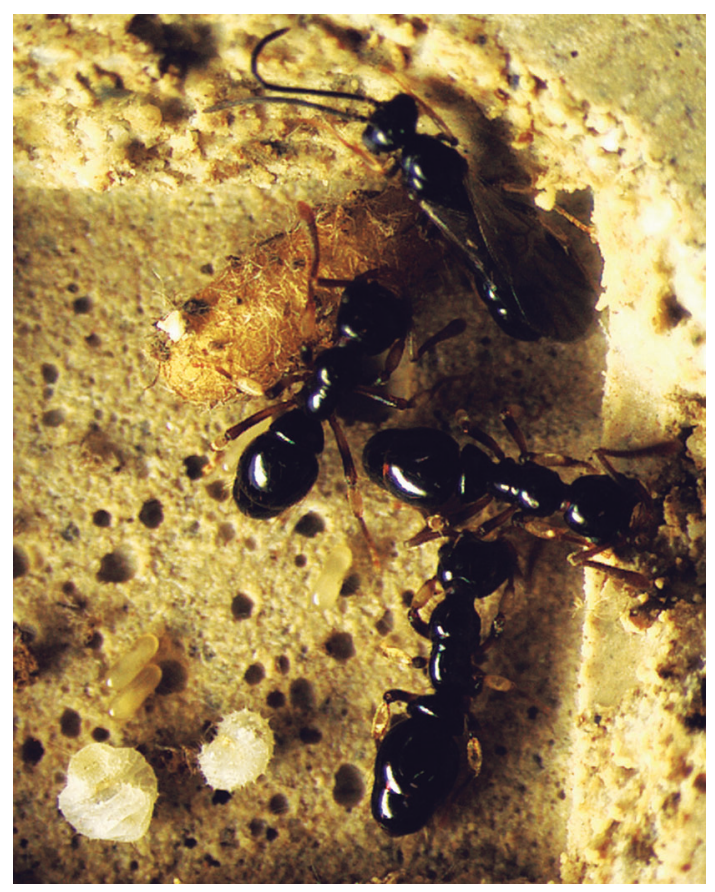

FIGURA 22.3 - Exemplos de sítios de nidificação de Thaumatomyrmex spp. em cavidades: a) casulos secos de Lepidoptera Limacodidae colados ao tronco de uma árvore (Foz do Iguaçu, PR), b) cupinzeiro terrícola (Rio Claro, SP), c) ninho de vespa solitária terrícola (Ilhéus, BA), d) concha do gastrópode terrestre Megalobulimus gummatus (Hidalgo, 1870) (Ilhéus, BA), e) Endocarpos de Dipteryx panamensis (Pittier) Record; Mell (Fabaceae) (Estacíon Biologica La Selva, Costa Rica)

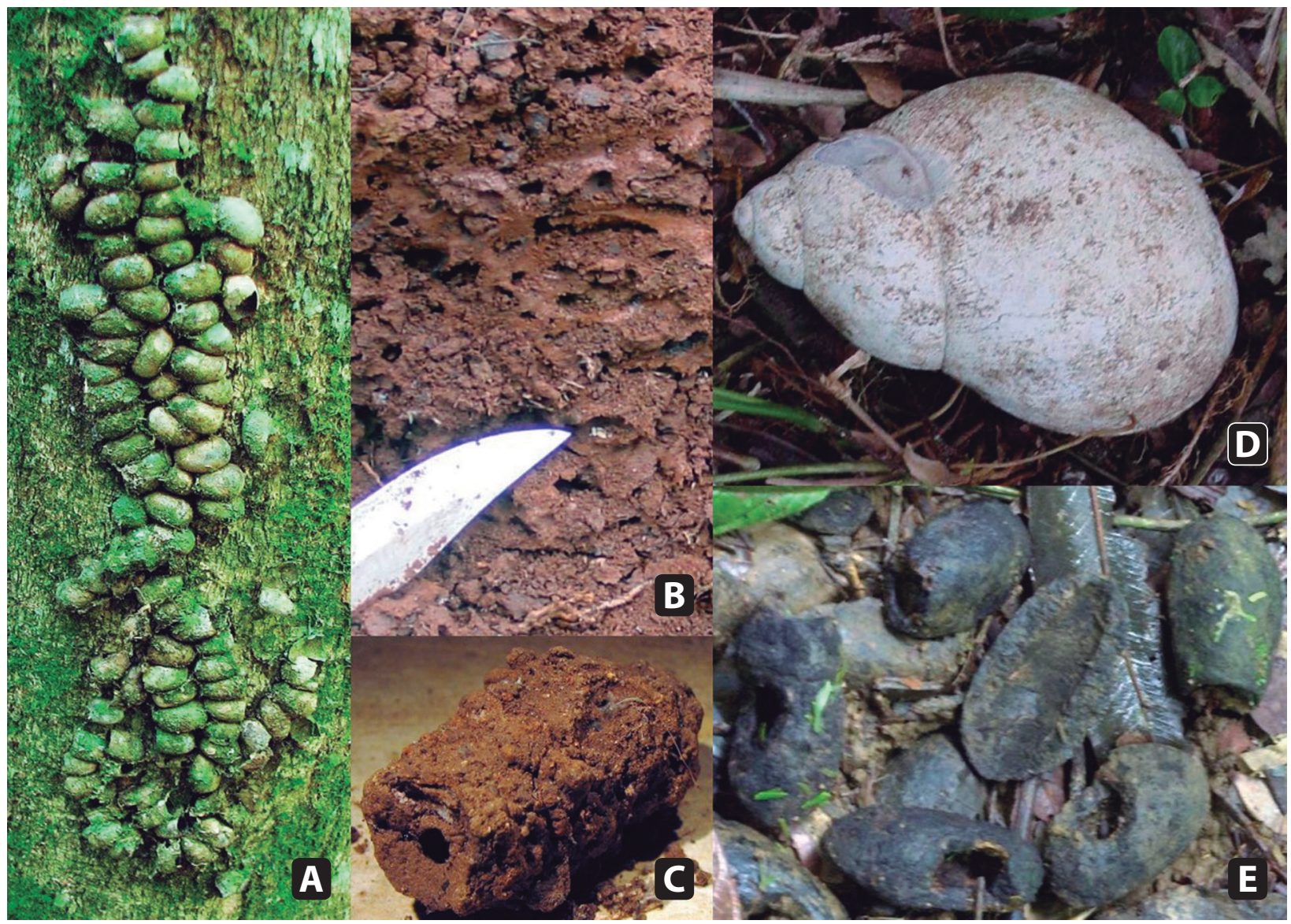


FIGURA 22.4 - Predação de um Penicillata (Monographis tamoyoensis Schubart, 1939; Polyxenida, Polyxenidae) por uma fêmea de Thaumatomyrmex contumax (Ilhéus, BA): a: captura; b: ferroada; c: transporte; d: inicio da sequência de retiradas do tricomas.

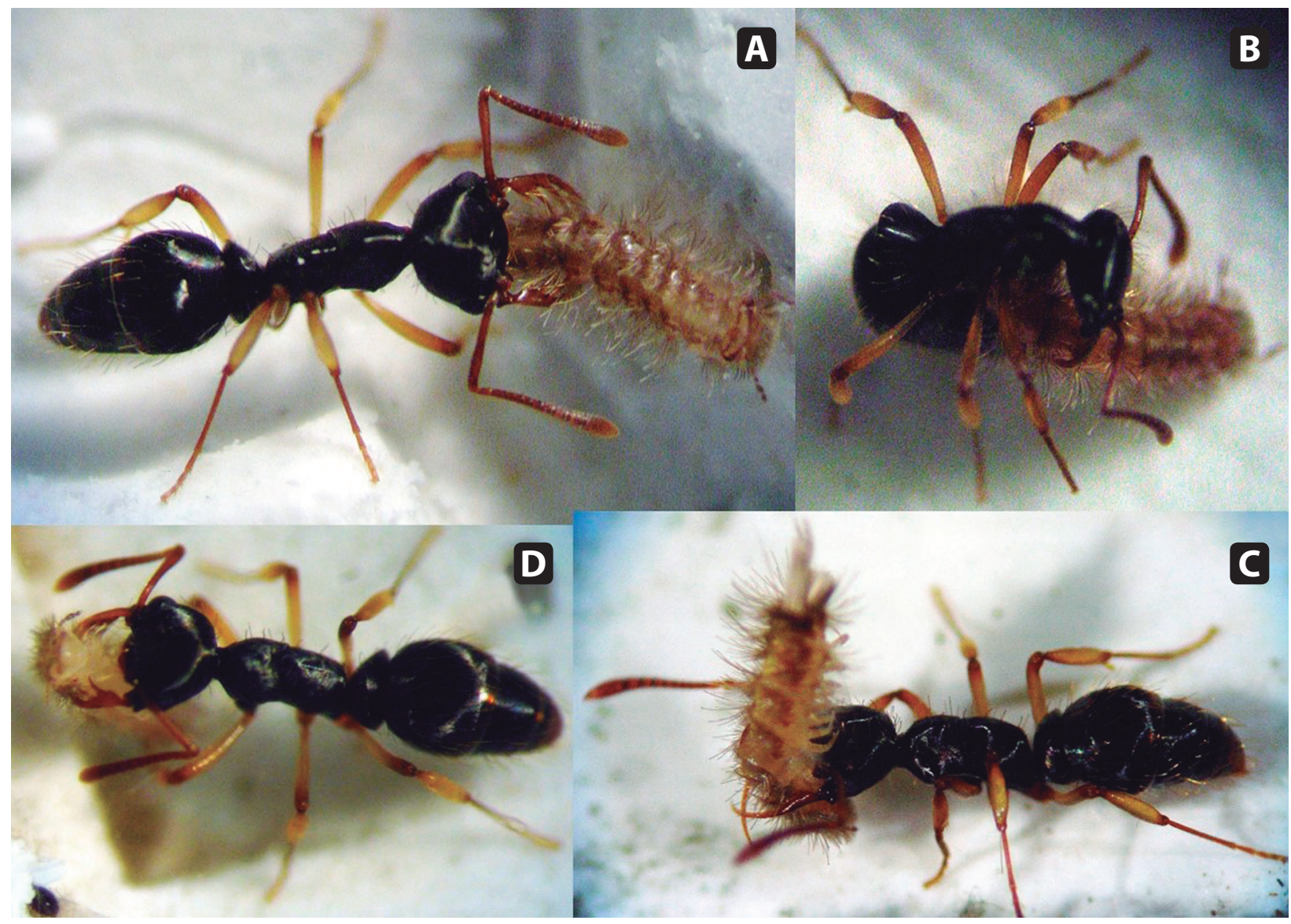

Thaumatomyrmex pode fornecer subsídios para a compreensão da evolução dos biomas desta região. Por exemplo, essas formigas se prestam à interpretação das consequências das oscilações paleoclimáticas do quaternário que refletiram nas fases alternadas de expansão e regressão de florestas úmidas (incluindo a Floresta Amazônica e a Mata Atlântica) e da vegetação aberta (Cerrado e Caatinga). Uma vez que essas formigas possuem características muito peculiares de nidificação e alimentação (BRANDÃO et al., 1991; JAHYNY et al., 2008, 2009; JAHYNY 2010), além de uma baixa capacidade de dispersão para a maioria das espécies (quando não existe a fêmea alada), espera-se que as populações dessas formigas respondam a variações climáticas amplas, assim como às mudanças rápidas da qualidade de seu habitat. Thaumatomyrmex parece ser, assim, um modelo ideal para abordar estudos sobre a formação da biodiversidade neotropical, ou em biomonitoramento a longo prazo.

\section{Metodologia}

Os dados de distribuição das espécies de Thaumatomyrmex que utilizamos resultam da compilação da literatura, do material visto pelos autores nas coleções CPDC, MZSP e INBio [ver EVENHUIS (2015) para a abreviação das coleções entomológicas indicadas neste trabalho] e de informações da Internet (AntWeb). Nos casos em que as informações relativas à localidade de coleta não tenham sido apropriadamente detalhadas na literatura ou nos rótulos, essas foram buscadas com o auxílio do GoogleEarth. Mapas de distribuição foram produzidos com o auxílio do software ArcGis 9.3. (ESRI 2009). A classificação dos climas segue Köppen (1936). Seguimos o agrupamento de espécies proposto por Kempf (1975), sendo que utilizamos as mesmas características definidas por esse autor para agrupar táxons encontrados ou descritos posteriormente nos grupos Ferox, Mutilatus e Cochlearis. 
TABELA 22.I - Espécies descritas e não descritas do gênero Thaumatomyrmex Mayr, 1887, classificadas de acordo com os grupos definidos por KEMPF (1975), e sua distribuição por país baseada em dados de literatura e coleções museológicas

\begin{tabular}{|c|c|c|c|}
\hline $\begin{array}{c}\text { Nome do agrupamento } \\
\text { de espécies }\end{array}$ & Espécie & Fonte das informações & Distribuiç̧ão (país) \\
\hline \multirow{6}{*}{ Grupo Cochlearis } & $\begin{array}{l}\text { Thaumatomyrmex bariay Fontenla } \\
\text { Rizo, } 1995\end{array}$ & $\begin{array}{l}\text { FONTENLA, RIZO 1995; BARONI } \\
\text { URBANI; DE ANDRADE, } 2003\end{array}$ & Cuba \\
\hline & $\begin{array}{l}\text { Thaumatomyrmex cochlearis } \\
\text { Creighton, } 1928\end{array}$ & $\begin{array}{c}\text { CREIGHTON, 1928; KEMPF 1975; } \\
\text { BARONI URBANI; DE ANDRADE, } \\
2003\end{array}$ & Cuba \\
\hline & $\begin{array}{l}\text { Thaumatomyrmex mandibularis } \\
\text { Baroni Urbani; De Andrade, } 2003\end{array}$ & $\begin{array}{c}\text { BARONI URBANI; DE ANDRADE, } \\
2003\end{array}$ & Cuba \\
\hline & $\begin{array}{l}\text { Thaumatomyrmex nageli Baroni } \\
\text { Urbani; De Andrade, } 2003\end{array}$ & $\begin{array}{l}\text { BARONI URBANI; DE ANDRADE， } \\
2003\end{array}$ & Cuba \\
\hline & Thaumatomyrmex sp.12 & AntWeb (casent0010674- UCDC) & $\begin{array}{l}\text { República } \\
\text { Dominicana }\end{array}$ \\
\hline & Thaumatomyrmex sp.13 & AntWeb (casent0173033 - MCZC) & $\begin{array}{l}\text { República } \\
\text { Dominicana }\end{array}$ \\
\hline \multirow{2}{*}{ Grupo Mutilatus } & $\begin{array}{l}\text { Thaumatomyrmex contumax Kempf, } \\
1975\end{array}$ & KEMPF, 1975; CPDC & Brasil \\
\hline & $\begin{array}{l}\text { Thaumatomyrmex mutilatus Mayr, } \\
1887\end{array}$ & $\begin{array}{l}\text { MAYR, 1887; KEMPF, 1975, CPDC; } \\
\text { MZUSP }\end{array}$ & $\begin{array}{l}\text { Argentina, Brasil, } \\
\text { Paraguai }\end{array}$ \\
\hline \multirow{22}{*}{ Grupo Ferox } & Thaumatomyrmex atrox Weber, 1939 & $\begin{array}{l}\text { WEBER, 1939; KEMPF, 1975; } \\
\text { CPDC; INBio; MCZ; AntWeb } \\
\text { (casent0010857 - UCDC); B. } \\
\text { Guénard com. pers. } 2008\end{array}$ & $\begin{array}{l}\text { Colômbia, Costa } \\
\text { Rica, Guiana, } \\
\text { Panama, Trinidad-e- } \\
\text { Tobago (apenas na } \\
\text { ilha de Trinidade), } \\
\text { Venezuela }\end{array}$ \\
\hline & Thaumatomyrmex ferox Mann, 1922 & MANN, 1922; KEMPF, 1975; & Honduras \\
\hline & Thaumatomyrmex manni Weber, 1939 & WEBER, 1939; ; KEMPF 1975 & Bolívia \\
\hline & $\begin{array}{l}\text { Thaumatomyrmex paludis Weber, } \\
1942\end{array}$ & $\begin{array}{l}\text { WEBER, 1942; KEMPF, 1975; } \\
\text { CPDC, H. Vasconcelos com. pers. } \\
\text { 2005; Vasconcelos 2008; AntWeb } \\
\text { (casent0173035 - MCZ) }\end{array}$ & Brasil, Venezuela \\
\hline & $\begin{array}{l}\text { Thaumatomyrmex soesilae Makhan, } \\
2007\end{array}$ & MAKHAN, 2007; CPDC & Brasil, Surinam \\
\hline & $\begin{array}{l}\text { Thaumatomyrmex zeteki Smith, M.R. } \\
1944\end{array}$ & SMITH, 1944; KEMPF 1975; CPDC & Colômbia, Panamá \\
\hline & Thaumatomyrmex sp.1 & CPDC & Brasil \\
\hline & Thaumatomyrmex sp.2 & CPDC & Brasil \\
\hline & Thaumatomyrmex sp.3 & $\begin{array}{c}\text { CPDC; AntWeb (casent0102935 - } \\
\text { BMNH); }\end{array}$ & $\begin{array}{l}\text { Brasil, Granada, } \\
\text { Guiana Francesa }\end{array}$ \\
\hline & Thaumatomyrmex sp.4 & AntWeb (casent0173031 - MCZC) & México \\
\hline & Thaumatomyrmex sp.5 & CPDC & Brasil \\
\hline & Thaumatomyrmex sp.6 & AntWeb (casent0178703 - MIZA) & Venezuela \\
\hline & Thaumatomyrmex sp.7 & CPDC & Guiana Francesa \\
\hline & Thaumatomyrmex sp.8 & CPDC & Brasil \\
\hline & Thaumatomyrmex sp.9 & CPDC & Colômbia \\
\hline & Thaumatomyrmex sp.10 & AntWeb (casent0010852 - UCDC) & Peru \\
\hline & Thaumatomyrmex sp.11 & CPDC, INBio, ALAS & $\begin{array}{l}\text { Costa Rica, } \\
\text { Nicaragua }\end{array}$ \\
\hline & Thaumatomyrmex sp.11 & CPDC, INBio, ALAS & $\begin{array}{l}\text { Costa Rica, } \\
\text { Nicaráqua } \\
\end{array}$ \\
\hline & Thaumatomyrmex sp.14 & $\begin{array}{c}\text { <http://people.bu.edu/karitr/ } \\
\text { Species/Thaumatomyrmes\%20 } \\
\text { undetermined.html> (último } \\
\text { acesso: 19/08/2008). }\end{array}$ & Equador \\
\hline & Thaumatomyrmex cf. ferox & KEMPF, 1975 pp. 120-121 & Belize \\
\hline & Thaumatomyrmex sp.16 & AntWeb (casent0178702 - MIZA) & Venezuela \\
\hline & Thaumatomyrmex sp. cf. sp. 1 & CPDC & Brasil \\
\hline
\end{tabular}




\section{Distribuição geográfica}

Doze espécies são conhecidas até o momento e uma quinzena de táxons novos está à espera de ser descrita (Tabela 22.I). Estima-se que o gênero seja representado na realidade por cerca de trinta táxons atuais com sua ocorrência restrita à Região Neotropical.

Thaumatomyrmex é exclusivamente neotropical (sensu Morrone 2014), distribuído entre $22^{\circ} \mathrm{N}$ no Caribe (Cuba) ou $18^{\circ} \mathrm{N}$ no continente norte-americano (México) para os limites norte, a $29^{\circ} \mathrm{S}$ (Brasil) para seu limite sul, e encontra-se desde o nível do mar até altitudes superiores a 2.000 metros nas montanhas da Colômbia (JAHYNY et al., 2008). Espécies deste gênero já foram coletadas em todos os países latino-americanos, exceto El Salvador, Chile e Uruguai. Encontram-se também nas Grandes Antilhas, nas ilhas de Cuba e Hispaniola, assim como nas Pequenas Antilhas (Granada e Trinidad). O gênero Thaumatomyrmex faz parte dos 56 gêneros endêmicos da Região Neotropical (em 119 conhecidos, ou seja, 47\%) (BOLTON et al., 2006; RABELING et al., 2008).

\section{Thaumatomyrmex grupo Cochlearis}

(Tabela 22.I; Figura 22.5)

As quatro espécies formalmente descritas de Thaumatomyrmex do grupo Cochlearis vêm todas da ilha de Cuba (BARONI URBANI; DE ANDRA$\mathrm{DE}, 2003)$. Exemplares de espécies não descritas e que também pertencem a esse grupo foram encontradas na República Dominicana na ilha Hispaniola (AntWeb (casent0010674 - UCDC); AntWeb (casent0173033 - MCZC)). Nenhuma espécie deste grupo foi coletada até agora no Arquipélago das Bahamas ou nas Pequenas Antilhas onde, no entanto, existem espécies do grupo Ferox nas ilhas mais próximas ao continente sul-americano como Granada e Trinidad (KEMPF 1975; Antweb (casent0102935 - BMNH)). As Thaumatomyrmex do grupo Cochlearis são, portanto, endêmicas das Grandes Antilhas. Duas espécies desse grupo (Thaumatomyrmex mandibularis Baroni Urbani; De Andrade, 2003 e Thaumatomyrmex sp.13) foram encontradas a mais de 800 metros de altitude, e os demais táxons vivem abaixo de 200 metros.

\section{Thaumatomyrmex grupo Mutilatus \\ (Tabela 22.I; Figura 22.5)}

As duas espécies de Thaumatomyrmex do grupo Mutilatus têm uma distribuição restrita à
América do Sul entre $03^{\circ} \mathrm{S}$ e $29^{\circ} \mathrm{S}$, com ocorrência desde o nível do mar até $1.000 \mathrm{~m}$ de altitude. Essas formigas são conhecidas somente do Brasil, Argentina e Paraguai. No Brasil, elas foram coletadas nos estados da Bahia, Ceará, Espírito Santo, Goiás, Maranhão, Mato Grosso, Mato Grosso do Sul, Minas Gerais, Pará, Pernambuco, Rio de Janeiro, Rio Grande do Sul, São Paulo, Santa Catarina e Tocantins. Das espécies brasileiras do gênero, Thaumatomyrmex contumax Kemf, 1975 e Thaumatomyrmex mutilatus Mayr, 1887 são as únicas a viver na Caatinga, no Cerrado e no Pantanal, três biomas do tipo savana. Apesar de serem também encontradas em florestas úmidas, as espécies deste grupo parecem estar mais adaptadas a um clima árido e/ou áreas de vegetação aberta do que as outras espécies de Thaumatomyrmex. Sua cutícula, finamente estriada e menos brilhante do que as demais espécies do grupo Ferox é, talvez, uma forma de adaptação a este ambiente.

\section{Thaumatomyrmex grupo Ferox (Tabela 22.I; Figura 22.5)}

As espécies de Thaumatomyrmex do grupo Ferox têm uma distribuição estritamente Neotropical, sendo encontradas entre $18^{\circ} \mathrm{N}$ (México) e $19^{\circ} \mathrm{S}$ (Brasil), desde o nível do mar a mais de 2.000 metros de altitude. Espécimes já foram coletados no México e em todos os países da América Central, com a exceção de El Salvador. Na América do Sul já foram coletados na Colômbia, Venezuela, Guiana, Suriname, Guiana Francesa, Brasil (estados do Acre, Amazonas, Bahia, Minas Gerais, Pará, Paraíba, Rondônia e Sergipe), Equador, Peru e Bolívia. Estão presentes nas ilhas de Trinidad e Granada nas Pequenas Antilhas. Essas espécies distribuemse em áreas onde o clima é Af ou Am (Classificação de Köppen: clima tropical úmido, sem estação seca ou clima tropical úmido com 1-3 meses secos, respectivamente), apenas alguns pontos de ocorrência estão situados numa região de clima $A w$, no estado da Bahia. Essas espécies provavelmente não estão adaptadas a climas áridos e a vegetação aberta, ao contrário das espécies do grupo Mutilatus. A presença de populações de Thaumatomyrmex sp.1 em enclaves de floresta úmida no meio da Caatinga (=brejos do Nordeste) testemunha, sem dúvida, a expansão da floresta durante o período mais quente e úmido do Quaternário (ver TABARELLI; SANTOS 2004; PINHEIRO et al., 2007). Durante a regressão dessas florestas, ilhas de vegetação 
FIGURA 22.5 - Distribuição conhecida dos três grupos de espécies do gênero Thaumatomyrmex Mayr, 1887

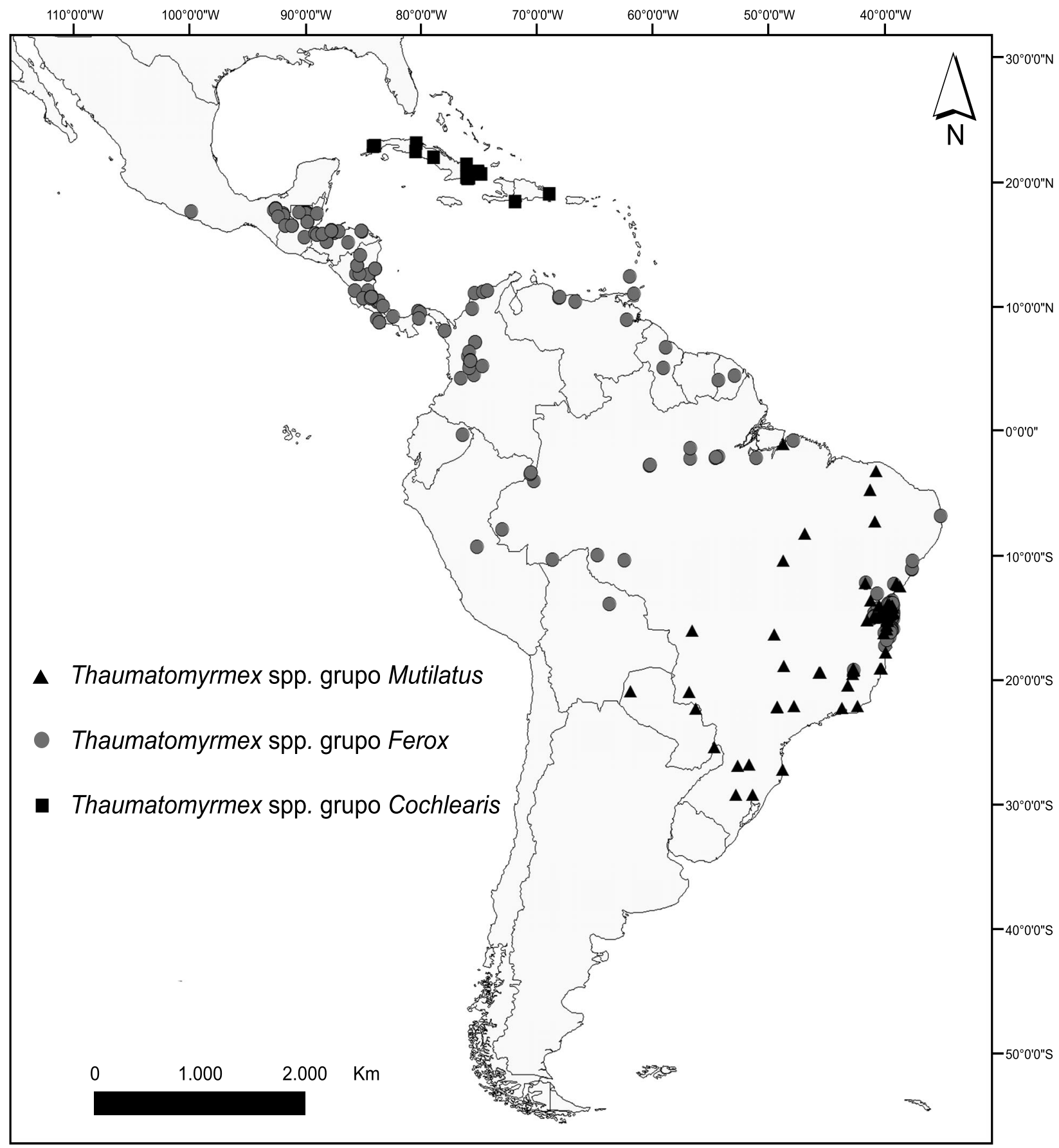

se mantiveram em áreas com micro-climas favoráveis, as quais podem ser consideradas refúgios para as espécies do bioma Mata Atlântica no meio da Caatinga (ver TABARELLI; SANTOS, 2004). Outros animais têm este tipo de distribuição descontínua, tais como alguns roedores (SOUSA et al., 2004). Com uma distribuição latitudinal que vai do México ao estado de Minas Gerais no Brasil, espécies do grupo Ferox representam possivelmente o grupo mais basal do gênero, mas somente estudos filogenéticos poderiam comprovar isso.
Na América do Sul, durante o Quaternário, os períodos frios e secos causaram uma expansão das áreas de savana para a região amazônica acompanhada por uma regressão e fragmentação da floresta úmida em áreas mais altas; estes períodos frios alternaram-se com períodos quentes e úmidos durante os quais a floresta úmida teria se expandido novamente enquanto a savana teria se recolhido. Populações disjuntas sobrevivendo nos refúgios teriam, então, se diferenciado por vicariância, tanto em refúgios de floresta úmida quanto de savana 
mais seca, em função do período climático e conforme a "Teoria dos refúgios quaternários" (HAFFER, 1969; PRANCE, 1973; HAFFER, 1997; MAYLE et al., 2000; HAFFER; PRANCE, 2001; PENNINGTON et al., 2004; CARNAVAL; MORITZ, 2008; HAFFER, 2008). As mudanças cíclicas de clima-vegetação do final do Cenozoico são consideradas por alguns como um dos principais fatores que levaram ao processo de especiação e diversificação das espécies de florestas e savanas tropicais da América do Sul (PENNINGTON et al., 2004; HAFFER, 2008). Mesmo se as mudanças climáticas cíclicas do Quaternário e, consequentemente, da vegetação, não tenham significativamente contribuído para a diversidade do gênero Thaumatomyrmex, elas tiveram certamente uma grande influência sobre a distribuição moderna das espécies. Isto pode também ter acontecido na ilha de Cuba onde os acontecimentos climáticos do Quaternário em alguns momentos isolaram ou limitaram as conexões entre as diferentes regiões da ilha por causa de inundações (ITURRALDE-VINENT, 2005).

\section{Organização simpátrica de algumas espécies de Thaumatomyrmex}

O estudo da distribuição das espécies permitiu destacar um fenômeno notável quanto à distribuição das espécies do gênero Thaumatomyrmex que, muitas vezes, são encontradas em pares simpátricos. $\mathrm{O}$ fato de que elas são, a priori, todas especializadas num único tipo de presas, os Diplopoda Penicillata, que não são muito diversificados morfologicamente, deve limitar suas possibilidades de ocupação dos estratos disponíveis no mesmo habitat. Procurou-se identificar algumas características destas espécies simpátricas e um sucinto estudo biométrico das espécies encontradas em simpátria foi realizado (isso não inclui espécies de Thaumatomyrmex do grupo Cochlearis para as quais não há dados disponíveis) (Tabela 22.II, Figura 22.6).

Existem várias combinações de pares de espécies simpátricas no gênero Thaumatomyrmex. Um determinado habitat não parece comportar mais do que duas espécies do gênero. Algumas características fazem dessas formigas modelos interessantes e simples para estudar essas simpatrias: são poucas espécies envolvidas; os ecossistemas implicados são bastante diversos; todas elas possuem provavelmente uma dieta similar baseada em Penicillata que são suas presas habituais. Como tal, elas formam a guilda de formigas predadoras especialistas de Penicillata. Estes Diplopoda possuem uma forma tubular em todos os estágios da sua vida, seu tamanho aumenta progressivamente até

TABELA 22.II - Algumas características biométricas dos pares de espécies simpátricas. Medidas e índices determinados a partir de um único indivíduo: TL: comprimento do corpo - HF: forma da cápsula cefálica (Q: Quadrada - A: Alongada (retangular) - T: Trapezoídeal (mais larga na frente)) - ML/HW: comprimento das mandíbulas em relação à largura da cabeça (NU: não ultrapassa o ângulo anterolateral da cabeça - U: ultrapassa o ângulo anterolateral da cabeça - HW: largura da cabeça na frente aos olhos - ML: comprimento mandibular - RTL: razão (TL espécie grande / TL espécie pequena) - RHW: razão (HW espécie grande / HW espécie pequena) - RML: razão (ML espécie grande / ML espécie pequena)

\begin{tabular}{|c|c|c|c|c|c|c|c|c|c|c|c|c|c|}
\hline \multirow{2}{*}{$\begin{array}{c}\text { Pequena/Grande espécies - } \\
\text { localidade }\end{array}$} & \multicolumn{5}{|c|}{ Pequena espécie } & \multicolumn{5}{|c|}{ Grande espécie } & \multirow[b]{2}{*}{ RTL } & \multirow[b]{2}{*}{ RHW } & \multirow[b]{2}{*}{ RML } \\
\hline & $\begin{array}{c}\mathrm{TL} \\
(\mathrm{mm})\end{array}$ & $\mathrm{HF}$ & $\begin{array}{l}\mathrm{ML} / \\
\mathrm{HW}\end{array}$ & $\begin{array}{l}\mathrm{HW} \\
(\mathrm{mm})\end{array}$ & $\begin{array}{c}\mathrm{ML} \\
(\mathrm{mm})\end{array}$ & $\begin{array}{c}\mathrm{TL} \\
(\mathrm{mm})\end{array}$ & $\mathrm{HF}$ & $\begin{array}{l}\mathrm{ML} / \\
\mathrm{HW}\end{array}$ & $\begin{array}{l}\mathrm{HW} \\
(\mathrm{mm})\end{array}$ & $\begin{array}{c}\mathrm{ML} \\
(\mathrm{mm})\end{array}$ & & & \\
\hline $\begin{array}{l}\text { Thaumatomyrmex sp.1 - T. contumax } \\
\text { - Brasil (CEPLAC, Ilhéus-BA) }\end{array}$ & 2,8 & $\mathrm{Q}$ & $\mathrm{NU}$ & 0,6 & 0,6 & 4,7 & $\mathrm{~T}$ & $U$ & 1,1 & 1,3 & 1,7 & 1,9 & 2,3 \\
\hline $\begin{array}{l}\text { Thaumatomyrmex sp.2 - T. } \\
\text { contumax - Brasil (Itacaré-BA) }\end{array}$ & 2,7 & $A$ & $\mathrm{NU}$ & 0,5 & 0,6 & 4,7 & $\mathrm{~T}$ & $U$ & 1,1 & 1,3 & 1,7 & 2,1 & 2,2 \\
\hline $\begin{array}{l}\text { Thaumatomyrmex sp.1 - T. mutilatus } \\
\text { - Brasil (Serra Campo Formoso ou } \\
\text { Olho D'água-Itambé-BA) }\end{array}$ & 2,8 & Q & $\mathrm{NU}$ & 0,6 & 0,6 & 4,1 & $\mathrm{Q}$ & NU & 1,0 & 1,0 & 1,5 & 1,8 & 1,8 \\
\hline $\begin{array}{l}\text { T. mutilatus - T. contumax - Brasil } \\
\text { (Olivença, Ilhéus-BA) }\end{array}$ & 3,4 & $\mathrm{Q}$ & NU & 0,7 & 0,7 & 4,6 & $\mathrm{~T}$ & $U$ & 1,2 & 1,3 & 1,4 & 1,7 & 1,8 \\
\hline $\begin{array}{l}\text { Thaumatomyrmex sp.11 - T. atrox } \\
\text { - Costa Rica (Estación Biológica La } \\
\text { Selva-Heredia) }\end{array}$ & 3,5 & $\mathrm{Q}$ & $\mathrm{NU}$ & 0,8 & 0,8 & 5,2 & $\mathrm{~T}$ & U & 1,4 & 1,6 & 1,5 & 1,9 & 2,1 \\
\hline $\begin{array}{l}\text { T. zeteki - T. atrox - Panama (Barro } \\
\text { Colorado) }\end{array}$ & 3,3 & $\mathrm{Q}$ & $\mathrm{NU}$ & 0,7 & 0,6 & 5,5 & $\mathrm{~T}$ & $U$ & 1,3 & 1,4 & 1,7 & 2,0 & 2,2 \\
\hline
\end{tabular}


FIGURA 22.6 - Relações alométricas entre os pares de espécies simpátricas no gênero Thaumatomyrmex. Os dados foram retirados da Tabela 22.II. As elipses delimitam separadamente as espécies de tamanhos pequeno e grande dos diferentes pares, respectivamente. Cada par é indicado pelo mesmo símbolo

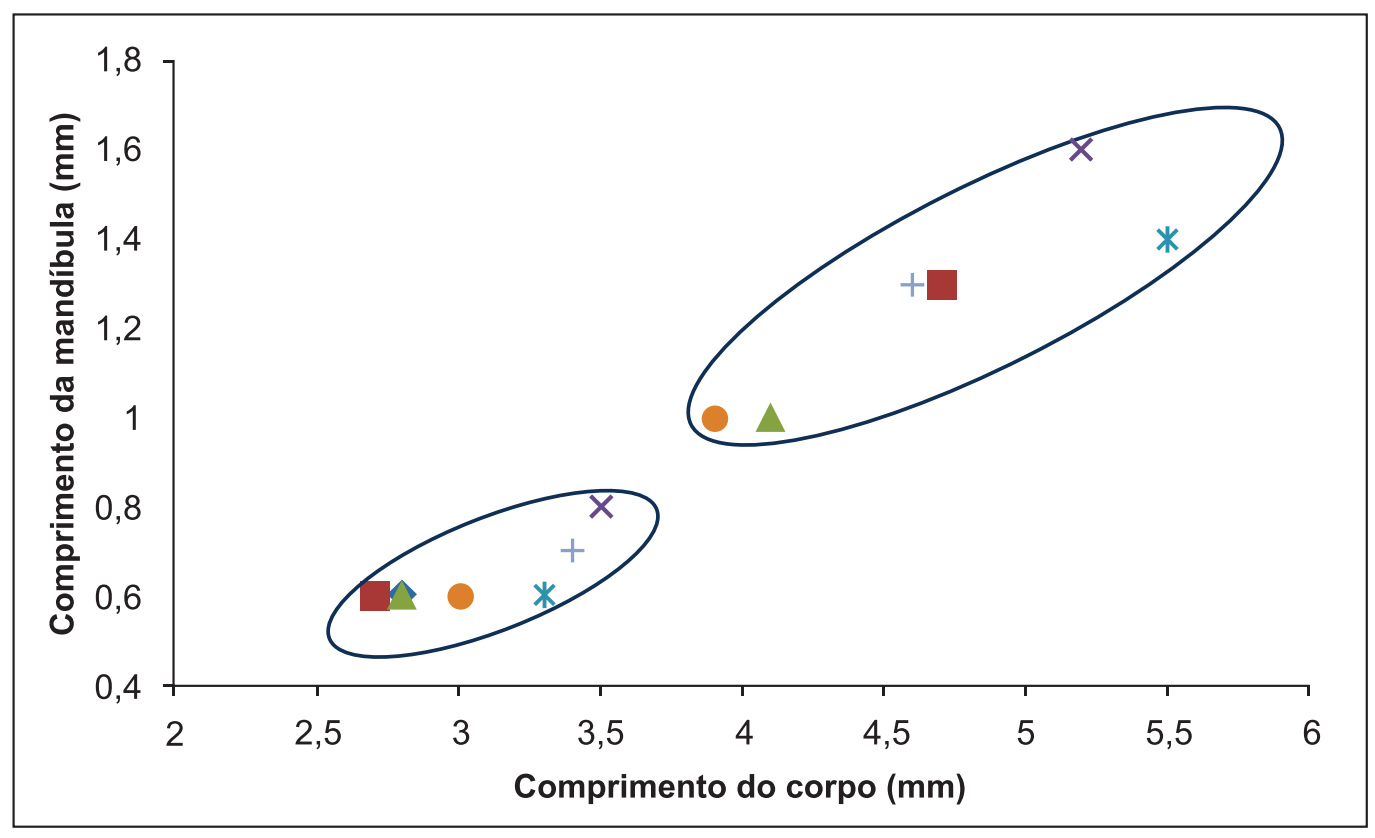

a idade adulta (onde atingem cerca de $5 \mathrm{~mm}$ ) e vivem em todos os estratos desde o solo até o dossel. O comprimento do corpo das Thaumatomyrmex, o das suas mandíbulas, além da largura da cabeça à frente dos olhos, são provavelmente determinantes na sua capacidade e eficácia para caçar os Penicillata de dada faixa de tamanho. Duas espécies de Thaumatomyrmex de mesmo tamanho dificilmente coexistiriam uma vez que iriam competir pelo mesmo tipo de recurso (por exemplo, Penicillata da mesma faixa de tamanho) ou locais de nidificação. As simpatrias conhecidas entre espécies de Thaumatomyrmex consistem sempre de uma espécie grande e de outra menor e, por isso, permitem a cada uma o acesso diferenciado aos recursos alimentares e aos locais de nidificação. Dados de coleta de ninhos e dados de criação de colônias no laboratório de duas espécies simpátricas, Thaumatomyrmex sp.1, a menor, e T. contumax, a maior, indicam que Thaumatomyrmex sp. 1 dificilmente consegue predar os Penicillata de um tamanho maior do que o dela e não utiliza conchas de Gastropoda de maior tamanho para nidificar, ao contrário de T. contumax que caça facilmente estes Penicillata grandes e não utiliza as conchas pequenas (JAHYNY, 2010). A diferença de tamanho é, muito possivelmente, um dos fatores que permitem a coexistência das espécies, reduzindo a sobreposição de seus respectivos nichos (WILSON, 1975). No caso de espécies filogeneticamente próximas, esse deslocamento de caracteres entre pares de espécies aumenta ainda a incompatibilidade sexual entre ambas e, assim, reduz a probabilidade de interações reprodutivas que representam um dos principais problemas para a coexistência de dois táxons aparentados (ver PFENNIG; PFENNIG 2009). Esse deslocamento de caracteres tem, assim, a propriedade de reduzir a probabilidade de ocorrer acasalamentos interespecíficos e hibridização, além de limitar a competição por recursos; ele pode ser de origem morfológica, ecológica, fisiológica ou comportamental (BROWN; WILSON, 1956; PFENNIG; PFENNIG, 2009). Este fenômeno encontra todo seu significado com a espécie $T$. mutilatus que, encontrada em simpatria com uma espécie maior, T. contumax, possui um tamanho menor do que quando encontrada em simpatria com uma espécie menor, Thaumatomyrmex sp.1. Além disso, vários casos de parapatria entre espécies de tamanhos equivalentes foram registrados no Brasil. Isto inclui tanto espécies presentes em áreas de mata contínua quanto nos fragmentos de mata distintos. Porém, em muitos casos, os dados de coleta são imprecisos e, por isso, novas coletas seriam necessárias para definir melhor os limites de distribuição de cada espécie. 
A coexistência de espécies que pertencem à mesma guilda, em geral, está baseada na diferenciação morfológica ligada ao tamanho do corpo ou de estruturas em conexão com a utilização do recurso na qual a guilda está baseada (HUTCHINSON, 1959). Esta diferenciação morfológica pode ser quantificada para espécies simpátricas potencialmente em competição, pela determinação do tamanho do corpo ou por características morfológicas relacionadas com a obtenção de alimentos (HUTCHINSON, 1959). Os resultados aqui apresentados são uma reminiscência da sugestão de Hutchinson (1959) segundo a qual, pares de espécies simpátricas muitas vezes diferem fortemente em tamanho. Hutchinson (1959) chegou a sugerir uma razão de tamanho mínimo de aproximadamente 1,3 que permitiria que duas espécies co-ocorressem em diferentes nichos, mas no mesmo nível da cadeia alimentar. Esse valor é amplamente ultrapassado nas simpatrias observadas no gênero Thaumatomyrmex (Tabela 22.II). As espécies de Thaumatomyrmex poderiam representar um dos raros casos conhecidos de similaridade limitante em formigas (ver FOITZIK; HEINZE, 1999) e até em insetos (ver PEARSON, 1980). A similaridade limitante é um conceito da ecologia teórica e ecologia de comunidades que propõe a existência de um nível máximo de sobreposição de nicho entre duas espécies permitindo uma coexistência contínua. A competição é normalmente menos intensa quanto maior é a diferença fenotípica. As espécies de Thaumatomyrmex de tamanho maior, frequentemente, possuem uma cabeça trapezoidal, alargada na frente, enquanto as espécies pequenas têm sua cápsula cefálica tão comprida quanto larga. Foi encontrada uma correlação significativa positiva entre a razão (comprimento / largura da cápsula cefálica) e o tamanho do corpo sem a cabeça (coeficiente de correlação de Pearson $=0,53 ; \mathrm{p}=$ 0,0001). A relação entre a morfologia de uma espécie e o tipo predominante de presa precisa ainda ser definida, a fim de compreendermos em maiores detalhes as relações entre espécies simpátricas através da morfologia.

\section{Distribuição diferenciada das espécies de Thaumatomyrmex com gamergates e com gines}

Doze espécies do gênero Thaumatomyrmex foram descritas, mas o gênero pode contar cerca de trinta espécies (JAHYNY et al., 2008; B. JAHYNY obs. pess.). A descrição das fêmeas era baseada unicamente em operárias até a descrição recente de uma gine identificada como Thaumatomyrmex ferox Mann, 1922 (VAZQUEZ et al., 2010). Até onde sabemos, existem em coleções mirmecológicas 11 espécimes de gines de pelo menos duas espécies diferentes de Thaumatomyrmex (Tabela 22.III, Figura 22.8), e o registro mais antigo foi mencionado por Kempf, em 1975 (D.R. SMITH, in litt.). Uma destas espécies com gine já é conhecida: Thaumatomyrmex zeteki M.R. SMITH, 1944; a outra é uma espécie ainda não descrita do oeste do Brasil depositada na coleção CPDC (Thaumatomyrmex sp.8). Gines e operárias de Thaumatomyrmex sp.8 (Figura 22.7) foram coletadas nas mesmas localidades em seis oportunidades. Suspeita-se que a gine observada por Vazquez et al. (2010) pertença à espécie Thaumatomyrmex zeteki, e não à Thaumatomyrmex ferox, em razão de suas características morfológicas, e por ter sido coletada no Panamá onde outras gines de T. zeteki já foram encontradas (coleção CPDC). Além destes dados, na página antweb.org, existem imagens de uma gine e de uma operária, provavelmente de uma mesma espécie ainda não identificada. Esses indivíduos encontram-se na coleção CASC, sem nenhuma informação de coleta (AntWeb - casent0006627 e casent0006628).

A coleta de ninhos sugere que o morfotipo gine é ausente em pelo menos sete espécies de Thaumatomyrmex: Thaumatomyrmex atrox Weber, 1939 (Panamá - Jonathan Shik com. pess. 2006), T. ferox (KEMPF, 1975), Thaumatomyrmex sp.1 (Brasil - JAHYNY, 2010), Thaumatomyrmex sp.2 (Brasil - JAHYNY, 2010), Thaumatomyrmex sp.11 (Costa Rica - JAHYNY, 2010), T. contumax (Brasil - JAHYNY, 2010) e T. mutilatus (Brasil JAHYNY, 2010). A dissecação de operárias coletadas em vários ninhos das cinco últimas espécies citadas mostrou que, dentro de cada colônia, pelo menos uma fêmea era fecundada e possuia a espermateca repleta de espermatozoides, ovaríolos com ovócitos em desenvolvimento e corpos amarelos, indicando que uma possível oviposição ocorreu. Estas espécies adotaram uma estratégia de reprodução através de "gamergates" (JAHYNY, 2010).

Sete gines de T. zeteki foram coletadas em três áreas de florestas tropicais pluviais no Panamá (Tabela 22.III). Esta espécie também está presente na província de Cundinamarca na Colômbia (KEMPF 1975). Três gines de Thaumatomyrmex sp. 8 foram encontradas no bioma amazônico (Tabela 22.III Figura 22.7). Uma das gines foi capturada em uma 
FIGURA 22.7 - Gine que perdeu as asas $(\mathrm{a}, \mathrm{b})$ e operária $(\mathrm{c}, \mathrm{d})$ de Thaumatomyrmex sp.8, cabeça em vista frontal $(\mathrm{a}, \mathrm{c})$ e vista lateral (b,d) (ESTEX-CEPLAC, Ouro Preto do Oeste, Rondônia); Estereomicroscópio LEICA M165C, Automontage, imagem: Odair Rodrigues. Fontes, CPDC

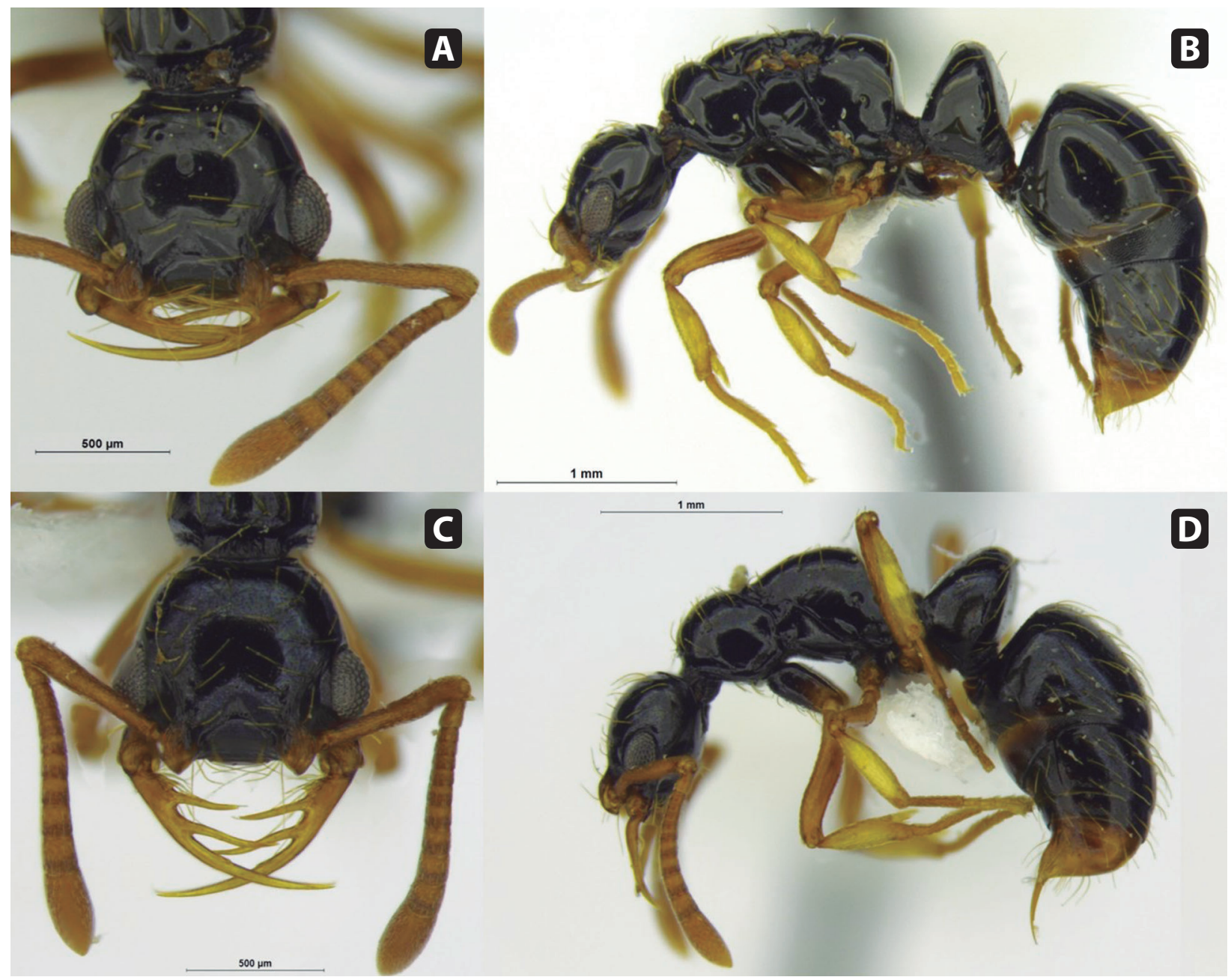

armadilha de queda; ela estava provavelmente forageando na serrapilheira em busca de alguma presa ou de um sítio de nidificação.

Gines aladas, energeticamente mais custosas de se produzir do que as operárias, são consideradas a forma de dispersão a longa distância, enquanto uma estratégia de reprodução com gamergates implica uma dispersão a curta distância, via fissão da colônia. Nesse caso, algumas operárias se separam do "ninho-mãe" para fundar uma nova colônia (ver PEETERS, 2012). Fatores ambientais (clima e tipo de vegetação) poderiam ter influência nas estratégias reprodutivas de uma espécie (colônias com gines ou com gamergates). No caso das Thaumatomyrmex, as gines das espécies com gines foram todas coletadas em área de floresta tropical pluvial. As espécies sem gine foram tanto encontradas em florestas tropicais pluviais quanto em áreas de vegetação mais aberta com clima semiárido como o Cerrado e a Caatinga, às quais elas estão bem adaptadas. Dados adicionais de coleta serão necessários para desvendar a exata influência dos fatores ambientais sobre a diferenciação dessas espécies.

\section{Hipótese biogeográfica sobre a radiação específica no gênero Thaumatomyrmex}

Como para as demais formigas (PEETERS, 2012), a presença de gine é uma característica plesiomórfica da subfamília Ponerinae, enquanto a condição presença de gamergates é apomórfica. Apesar do raro material biológico disponível, temos evidências que as poucas espécies de Thaumatomyrmex com gines pertencem todas ao grupo Ferox, enquanto para os demais grupos (Cochlearis e Mutilatus) e provavelmente para a maioria dos táxons do grupo Ferox, as espécies 
FIGURA 22.8 - Distribuição das espécies do gênero Thaumatomyrmex Mayr, 1887 sem gine e com gines

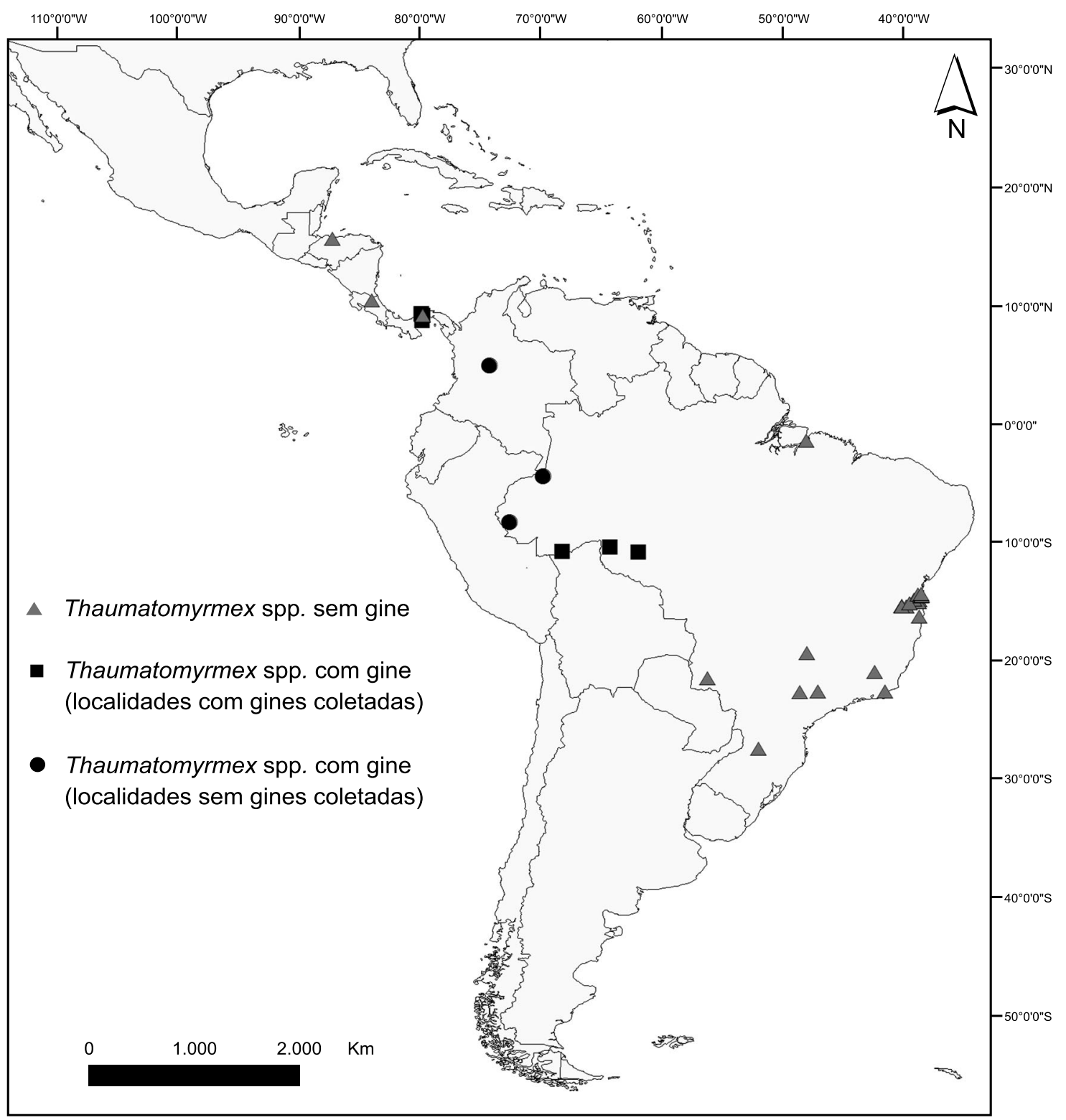

se reproduzem através da casta operária. Como já apontado, a ampla distribuição latitudinal do grupo Ferox, que vai do México ao estado de Minas Gerais no Brasil, sugere também que esse grupo (ou parte do mesmo) corresponde à linhagem mais basal do gênero. Isso indica, então, que o ancestral comum a todas as espécies atuais de Thaumatomyrmex tenha se originado na América Central ou no noroeste da Bacia Amazônica. Esta área coincide com a do gênero Belonopelta Mayr, 1870, que é um forte candidato a ser o grupo irmão de Thaumatomyrmex em estudos filogenéticos (ver SCHMIDT; SHATTUCK, 2014). Essa mesma região extrapola um pouco os limites para o sul e o leste daquela que Lattke (2003) caracteriza, e que é uma zona com grande endemicidade de Formicidae, onde se destacam os gêneros Lenomyrmex Fernández; Palacio, 1999 e Protalaridris Brown, 1980, e que corresponde às províncias Ocidente do Istmo do Panamá, Chocó, Magdalena, Cauca e Ocidente do Equador (MORRONE, 2001; LATTKE, 2003).

A origem monofilética das Thaumatomyrmex com gamergates é somente uma hipótese de trabalho nas condições de conhecimento atuais, mas poderia ser confirmada com o uso de ferramentas filogenéticas, além de mais informações sobre a biologia dessas formigas. Em razão de sua ampla distribuição e da quantidade de espécies com ou sem gamergates, a monofilia do grupo Ferox é bastante incerta. No entanto, se a origem das gamergates se deu 
TABELA 22.III - Dados de coleta das espécies de Thaumatomyrmex com gines

\begin{tabular}{|c|c|c|c|c|c|c|}
\hline Espécie & País & $\begin{array}{l}\text { Localidade e data de } \\
\text { coleta }\end{array}$ & $\begin{array}{l}\text { Técnica de } \\
\text { coleta }\end{array}$ & $\begin{array}{l}\text { Número } \\
\text { de gines } \\
\text { coletadas }\end{array}$ & $\begin{array}{c}\text { Número de } \\
\text { operárias } \\
\text { coletadas }\end{array}$ & $\begin{array}{c}\text { Fonte das } \\
\text { informações }\end{array}$ \\
\hline \multirow{3}{*}{$\begin{array}{c}\text { Thaumatomyrmex } \\
\text { zeteki M.R. SMITH, } \\
1944\end{array}$} & \multirow{3}{*}{ Panamá } & Ilha de Barro Colorado & $?$ & 1 & 0 & KEMPF 1975 \\
\hline & & $\begin{array}{l}\text { Colon - San Lourenzo } \\
\text { Forest - 30/12/2004 }\end{array}$ & $\begin{array}{l}\text { extrator } \\
\text { Winkler }\end{array}$ & 5 & 11 & CPDC \\
\hline & & $\begin{array}{c}\text { Cerro Campana - } \\
\text { 05/06/1995 }\end{array}$ & $\begin{array}{l}\text { extrator } \\
\text { Winkler }\end{array}$ & 1 & 1 & VAZQUEZ et al., 2010 \\
\hline $\begin{array}{c}\text { Thaumatomyrmex } \\
\text { sp. não } \\
\text { identificada }\end{array}$ & $?$ & $?$ & $?$ & 1 & 1 & $\begin{array}{c}\text { Antweb } \\
\text { casent0006627 e } \\
\text { casent0006628 - } \\
\text { CASC } \\
\end{array}$ \\
\hline \multirow{5}{*}{$\begin{array}{c}\text { Thaumatomyrmex } \\
\text { sp.8 (CPDC) }\end{array}$} & \multirow{4}{*}{ Brasil } & $\begin{array}{c}\text { Amazonas - Benjamin } \\
\text { Constant - Proj. BioBrasil/ } \\
\text { GEF/UNEP }\end{array}$ & $?$ & 0 & 8 & CPDC \\
\hline & & $\begin{array}{l}\text { Rondônia - Município } \\
\text { Ouro Preto do Oeste - } \\
\text { Estex (Estação da Ceplac) } \\
\text { - cacaual - 13/03/1998 }\end{array}$ & $\begin{array}{l}\text { extrator } \\
\text { Winkler }\end{array}$ & 1 & 1 & CPDC \\
\hline & & $\begin{array}{c}\text { Rondônia - Nova } \\
\text { Mamoré - Parque } \\
\text { Estadual Guajará Mirim - } \\
\text { 02/02/1998 }\end{array}$ & $\begin{array}{l}\text { armadilha } \\
\text { de queda } \\
\text { (pitfall traps) }\end{array}$ & 1 & 0 & CPDC \\
\hline & & $\begin{array}{l}\text { Acre - Porto Walter - } \\
05 / 02-17 / 04 / 1997\end{array}$ & $?$ & 0 & 1 & CPDC \\
\hline & Brasil & $\begin{array}{c}\text { Acre - RESEX Chico } \\
\text { Mendes Xapuri - } \\
\text { 14/10/2008 }\end{array}$ & $\begin{array}{l}\text { extrator } \\
\text { Winkler }\end{array}$ & 1 & 1 & Lacau S. com. pess. \\
\hline
\end{tabular}

numa população a partir da qual as espécies sem gamergates atuais do grupo Ferox se diferenciaram, hipotetiza-se que: 1) as populações encontradas na parte leste do território brasileiro derivam de uma colonização relativamente recente; 2) o atual grupo Cochlearis deriva da colonização das Grandes Antilhas por algum ancestral com gamergates do grupo Ferox e o grupo Cochlearis é muito provavelmente monofilético. Quanto ao grupo continental Mutilatus, as características morfológicas de suas espécies e sua distribuição relativamente limitada ao leste do Brasil até a Argentina e o Paraguai sugerem também sua monofilia e uma radiação no Cerrado e na Mata Atlântica.

A ocorrência de fêmeas reprodutoras sem asas é uma convergência entre espécies de vários gêneros e de várias subfamílias de Formicidae, portanto, as ligações com os parâmetros ecológicos e história de vida são múltiplas e variadas (PEETERS, 2012). Há pouco consenso na literatura sobre quais fatores ambientais favorecem uma fundação independente de colônia (principalmente via gines aladas) ou uma fundação dependente (o caso de espécies com gamergates) (PEETERS,
2012). O estudo do comportamento alimentar de várias espécies de Thaumatomyrmex indica que essas formigas têm como presas quase exclusivas os Penicillata (BRANDÃO et al., 1991; JAHYNY et al., 2008, 2009; JAHYNY, 2010; RABELING et al., 2012), que podem ser localmente muito abundantes e para as quais as Thaumatomyrmex não têm competidor conhecido na Região $\mathrm{Ne}$ tropical, da qual são endêmicas. As mandíbulas das Thaumatomyrmex não permitem a excavação, e todos os ninhos coletados foram encontrados em cavidades naturais (JAHYNY, 2010). Todas as colônias coletadas de várias espécies tinham poucas fêmeas por ninho, menos de cinco em geral (JAHYNY, 2010). Provavelmente, a maioria das Thaumatomyrmex têm em comum estas três características: predação de Penicillata, nidificação em cavidades naturais e colônias muito pequenas. Com isso, o gênero Thaumatomyrmex se torna um modelo muito particular e interessante para analisar a evolução das estratégias de reprodução que poderiam ser estudadas com a adição de informações sobre sua distribuição, ecologia e comportamento, assim como através de uma filogenia do gênero. 


\section{Referências}

AntWeb. Disponível em: <http://www.antweb.org>. Acesso em: 9 de março 2015

BARONI URBANI, C.; DE ANDRADE, M. L. The ant genus Thaumatomyrmex in Cuba (Hymenoptera: Formicidae) with description of two new species. Mittheilungen der Schweizer Entomologischen Gesellschaft, v. 76, p. 263-277, 2003.

BESTELMEYER, B. T., AGOSTI, D., ALONSO, L. E., BRANDÃO, C. R. F., BROWN JR., W. L., DELABIE, J. H. C.; SILVESTRE, R. Field techniques for the study of ground-living ants: an overview, description, and evaluation. In: Agosti, D.; Majer, J. D.; Tennant de Alonso; L.; Schultz, T. (Org.). Ants: Standard Methods for Measuring and Monitoring Biodiversity. Washington: Smithsonian Institution, 2000. p. 122-144.

BOLTON, B., ALPERT, G., WARD, P. S.; NASKRECKI, P. Bolton's catalogue of ants of the World: 1758-2005. CD-ROM. Cambridge: Harvard University Press. 2006.

BRANDÃO, C. R. F., DINIZ, J. L. M.; TOMOTAKE, E. M. Thaumatomyrmex strips millipedes for prey: a novel predatory behaviour in ants, and the first case of sympatry in the genus (Hymenoptera: Formicidae). Insectes Sociaux, v. 38, p. 335-344, 1991.

BROWN, W. L. Jr.; WILSON, E. O. Character displacement. Systematic Zoology, v. 5, n. 2, p. 49-64, 1956.

CARNAVAL, A. C.; MORITZ, C. Historical climate modelling predicts patterns of current biodiversity in the Brazilian Atlantic forest. Journal of Biogeography, v. 35, n. 7, p. 1187-1201, 2008.

DELABIE, J. H. C.; NASCIMENTO, I. C.; FONSECA, E. C.; SGRILLO, R. B. SOARES, P. A. O.; CASIMIRO, A. B.; FURST, M. Biogeografia das formigas cortadeiras (Hymenoptera; Formicidae; Myrmicinae; Attini) de importância econômica no leste da Bahia e nas regiões periféricas dos Estados vizinhos. Agrotrópica, v. 9, n. 2, p. 49-58, 1997.

DELABIE, J. H. C., FRESNEAU, D.; PEZON, A. Notes on the ecology of Thaumatomyrmex spp. (Hymenoptera: Formicidae: Ponerinae) in Southeast Bahia, Brazil. Sociobiology, v. 36, n. 3, p. 571-584, 2000.

DELABIE, J. H. C., JAHYNY B., NASCIMENTO, I. C., MARIANO, C. S. F., LACAU, S., CAMPIOLO, S., PHILPOTT, S. M.; LEPONCE, M. Contribution of cocoa plantations to the conservation of native ants (Insecta: Hymenoptera: Formicidae) with a special emphasis on the Atlantic Forest fauna of southern Bahia, Brazil. Biodiversity Conservation, v. 16, p. 2359-2384, 2007a.
DELABIE, J. H. C. D.; ALVES, H. S. R.; FRANÇA, V. C.; MARTINS, P. T. A.; NASCIMENTO, I. C. Biogeografia das formigas predadoras do gênero Ectatomma (Hymenoptera: Formicidae: Ectatomminae) no leste da Bahia e regiões vizinhas. Agrotrópica, v. 19, p. 13-20, 2007b.

DELABIE, J. H. C.; ALVES, H. S. R.; REUSSSTRENZEL, G. M.; CARMO, A. F. R.; NASCIMENTO, I. C. Distribuição das formigas cortadeiras dos gêneros Acromyrmex e Atta no Novo Mundo. In: Della Lucia, T.M.C. (Org.). Formigas Cortadeiras: da Bioecologia ao Manejo. Viçosa: Universidade Federal de Viçosa, 2011. p. 80-101.

ESRI 2009. ArcGIS Desktop: Release 9.3. Redlands, CA: Environmental Systems Research Institute

EVENHUIS, N. L. 2015. The insect and spider collections of the world website. Disponível em: $<$ http://hbs.bishopmuseum.org/codens/>. Acesso em: 13 abr. 2015.

FOITZIK, S.; HEINZE, J. Non-random size differences between sympatric Leptothorax ants. Entomologia Generalis, v. 24, p. 65-74, 1999.

HAFFER J. Speciation in Amazonian forest birds. Science, v. 165, p.131-137, 1969.

HAFFER, J. Alternative models of vertebrate speciation in Amazonia: an overview. Biodiversity and Conservation, v. 6, p. 451-476, 1997.

HAFFER, J. Hypotheses to explain the origin of species in Amazonia. Brazilian Journal of Biology, v. 68, n. 4, p. 917-947, 2008.

HAFFER, J.; PRANCE, G. T. Climatic forcing of evolution in Amazonia during the Cenozoic: on the refuge theory of biotic differentiation. Amazoniana, v. 16 , p. 579-607, 2001.

HUTCHINSON G. E. Homage to Santa Rosalia or why are there so many kinds of animals? American Naturalist, v. 93, p. 145-159, 1959.

ITURRALDE-VINENT, M. A. La paleogeografía del Caribe y sus implicaciones para la biogeografía histórica. Revista del Jardín Botánico Nacional, v. 2526, p. 49-78, 2005.

JAHYNY, B. Histoire naturelle du genre de fourmis néotropical Thaumatomyrmex Mayr 1887 (Arthropoda, Insecta, Hymenoptera, Formicidae, Ponerinae, Thaumatomyrmecini). 2010.582 f. Tese (Doutorado em Etologia). Université Paris XIII, Villetaneuse, 2010. 
JAHYNY, B., DELABIE, J. H. C.; FRESNEAU D. Mini-sociétés sans reine chez le genre neotropical Thaumatomyrmex Mayr, 1887 (Formicidae : Ponerinae). In: Actes des Colloques Insectes Sociaux, v. 15, 2002, Versailles.

JAHYNY, B.; LACAU, S.; DELABIE, J. H. C.; FRESNAU, D. 2008. Le genre Thaumatomyrmex Mayr 1887, cryptique et prédateur spécialiste de Diplopoda Penicillata. In: Jiménez, E., Fernández, F., Arias T. M.; Lozano-Zambrano, F. H. (Org.). Sistemática, Biogeografía y Conservación de las Hormigas Cazadoras de Colombia. Bogotá: Instituto de Investigación de Recursos Biológicos Alexander von Humboldt, 2008. p.329-346.

JAHYNY, B.; LACAU, S.; FRESNEAU, D.; DELABIE, J. H. C. Historia natural das formigas do gênero neotropical Thaumatomyrmex Mayr 1887 (Ponerinae, Thaumatomyrmecini). In: Simpósio de Mirmecologia, n. 19, 2009, Ouro Preto.

KEMPF, W. A revision of the Neotropical ponerine ant genus Thaumatomyrmex Mayr (Hymenoptera: Formicidae). Studia Entomologica, v. 18, p. 95-126, 1975.

KÖPPEN W. Das geographische System der Klimate. In: Köppen, W.; Geiger, R. (Org.) Handbuch der Klimatologie. Berlin: Gebrüder Borntraeger, 1936. p $1-44$.

KUSNEZOV, N. Zoogeografia de las hormigas en Sudamerica. Acta Zoologica Lilloana, v. 19, p. 25-186, 1963.

LATTKE, J. E. Biogeografía de las hormigas neotropicales. In: Fernández, F. (Org.). Introducción a las hormigas de la región Neotropical. Bogotá: Instituto de Investigación de Recurso Biológicos Alexander von Humboldt, 2003. p 65-85.

LATTKE, J. E. Retos biogeográficos de las hormigas del trópico Americano. Biológico, v. 69, suplemento 2, p. 213-216, 2007.

LONGINO, J. 1988. Notes on the taxonomy of the Neotropical ant genus Thaumatomyrmex Mayr (Hymenoptera: Formicidae). In: Trager, J. C.; Brill, E. J. (Org.). Advances in Myrmecology. New York: Leiden, 1988. p. 35-42.

LONGINO, J. T.; CODDINGTON, J.; COLWELL, R. $\mathrm{K}$. The ant fauna of a tropical rain forest: Estimating species richness three different ways. Ecology, v. 83, n. 3, p. 689-702, 2002.

MAYLE F.E., BURBRIDGE R.; KILLEEN T.J. Millennial-scale dynamics of southern Amazonian rain forests. Science, v. 290, p. 2291-2294, 2000.
MORRONE, J. J. Biogeografía de América Latina y el Caribe. Manuales y Tesis SEA, v. 3. 2001.

MORRONE, J. J. Biogeographical regionalisation of the Neotropical region. Zootaxa, v. 3782, p. 1-110, 2014.

PARR, C. L.; CHOWN, S. L. Inventory and bioindicator sampling: testing pitfall and Winkler methods with ants in a South African savanna. Journal of Insect Conservation, v. 5, p. 27-36, 2001.

PEARSON, D. L. Patterns of limiting similarity in tropical forest tiger beetles (Coleoptera: Cicindelidae). Biotropica, v. 12, p. 195-204, 1980.

PEETERS, C. Convergent evolution of wingless reproductives across all subfamilies of ants, and sporadic loss of winged queens (Hymenoptera: Formicidae). Myrmecological News, v. 16, p. 75-91, 2012.

PEETERS, C.; CREWE, R. Insemination controls the reproductive division of labour in a ponerine ant. Naturwissenschaften, v. 71, p. 50-51, 1984.

PENNINGTON, R. T.; LAVIN, M.; PRADO, D. E.; PENDRY, C. A.; PELL, S. K.; BUTTERWORTH, C. A. Historical Climate Change and Speciation: Neotropical Seasonally Dry Forest Plants show Patterns of both Tertiary and Quaternary Diversification. Philosophical Transactions of the Royal Society, London B, v. 359, p. 515-538, 2004.

PFENNIG, K. S.; PFENNIG, D. W. Character displacement: ecological and reproductive responses to a common evolutionary problem. Quarterly Review of Biology, v. 84, p. 253-276, 2009.

PINHEIRO, S. F.; OLIVEIRA, F. M. P.; CPRDEIRO, H. T. L.; QUINET, Y. Soil arthropod diversity in two moist montane forests of the Semi-Arid Brazilian. In: Congresso de Ecologia do Brasil, n. 8, 2007, Caxambu. Anais do VIII Congresso de Ecologia do Brasil. Caxambu: IBUSP. p. 1-2.

PRANCE, G. T. Phytogeographic support for the theory of Pleistocene forest refuges in the Amazon basin, based on evidence from distribution patterns in Caryocaraceae, Chrysobalanaceae, Dichapetalaceae and Lecythidaceae. Acta Amazonica, v. 3, p. 5-28, 1973.

RABELING, C.; BROWN, J. M.; VERHAAGH, M. Newly discovered sister lineage sheds light on early ant evolution. Proceedings of the National Academy of Sciences of the United States of America, v. 105, n. 39, p. 14913-14917, 2008. 
RABELING, C.; VERHAAGH, M.; GARCIA, M. V. B. Observations on the specialized predatory behavior of the pitchfork-mandibled ponerine ant Thaumatomyrmex paludis (Hymenoptera: Formicidae). Breviora, v. 533, p. 1-8, 2012.

SCHMIDT C. A.; SHATTUCK, S. O. The higher classification of the ant subfamily Ponerinae (Hymenoptera: Formicidae), with a review of ponerine ecology and behavior. Zootaxa, v. 3817, n. 1, p. 001-242, 2014.

SOUSA, M. A. N., LANGGUTH, A.; GIMENEZ, E. do A. Mamíferos dos Brejos de Altitude Paraíba e Pernambuco. In: Pôrto, K. C.; Cabral, J. J. P.; Tabarelli, M. (Org.). Brejos de Altitude em Pernambuco e Paraíba: História Natural, Ecologia e Conservação. Brasília: Ministério do Meio Ambiente, 2004. p. 229254.
TABARELLI, M.; SANTOS, A. M. M. Uma breve descrição sobre a história natural dos Brejos nordestinos. In: Pôrto, K. C.; Cabral, J. J. P.; Tabarelli, M. (Org.). Brejos de altitude em Pernambuco e Paraíba: História Natural, Ecologia e Conservação. Brasília: Ministério do Meio Ambiente, 2004. p. 17-24.

UNDERWOOD, E. C.; FISHER, B. L. The role of ants in conservation monitoring: If, when, and how. Biological Conservation, v. 132, p. 166-182, 2006.

VAZQUEZ, M; DASH; S. T.; MACKAY W. P. Description of the gyne of the ant Thaumatomyrmex ferox Mann, 1922 (Hymenoptera: Formicidae).

Entomologica Americana, v. 11, n. 3; 4, p. 25-28, 2010

WILSON, D. S. The adequacy of body size as a niche difference. The American Naturalist, v. 109, p. 769784, 1975. 